\title{
Reduction of discrete element models by Karhunen-Loève transform: a hybrid model approach
}

\author{
Philipp Glösmann
}

Received: 16 February 2009 / Accepted: 22 November 2009 / Published online: 19 December 2009

(c) The Author(s) 2009. This article is published with open access at Springerlink.com

\begin{abstract}
The term "discrete element method" (DEM) in engineering science comprises various approaches to model physical systems by agglomerates of free particles. While shapes, sizes and properties of particles may vary, in most DEM models, particles are not confined by constraints, but subject to applied forces derived from potential fields and/or contact laws. This general approach allows for widespread use of DEM models for physical phenomena including gas dynamics, granular flow, fracture and impact analysis. However, its characteristic feature, combining particle restraints and forces into applied forces, does not only provide for flexible adaption of DEM to different physics, but also creates the most limiting restriction: Evaluation of the applied forces for each particle is computational expensive restraining the time sequence and sample size for numerical analyses. As an ansatz to circumvent this obstacle for a class of DEM models, we propose a model order reduction method based on coherency in the dynamics of particles. While initial flexibility of DEM is conserved, computational effort can be reduced significantly.
\end{abstract}

Keywords Discrete element method - Karhunen-Loève transform $\cdot$ Model order reduction $\cdot$ Hybrid adaptive model

\section{Introduction}

The discrete element method (DEM), often also referred to as molecular dynamics (MD) or Distinct Element Method, has become a well established method for the numerical simulation of mechanical systems. Introduced by Cundall

P. Glösmann $(\varangle)$

Department of Mechanical Engineering, University of California, Berkeley, USA

e-mail: gloesmann@tu-harburg.de
[1] to investigate the dynamics of granular material, the DEM is currently used to model such phenomena as granular settlement, e.g. [2,3], stability of assemblies [4-7] or granular/structure interactions in material handling processes [8-10]. In recent publications the DEM is expanded to analyses of solid structures, ref. e.g. [11]. The research interest focuses on three main topics: impact, fracture and stress in homogeneous or compound material. Current studies cover impact behavior of automobile glass [12], fatigue or fracture of specimen under various stresses [13-16], and also stress in structures under large deformation [17] or loads [18].

Despite the multitude of applications, further spread of the DEM is restrictively coupled to progress in computational power: Typically, DEM models are based on the assumption of unrestraint single particles subject to potential fields. This approach allows for very simple formulations of the equations of motion (5) and (6) and thus for flexible models. However, the evaluation of the right-hand sides of Eqs. (5) and (6) can become very numerical expensive, depending on the range and choice of governing potentials $U$. Up to date, the numerical costs limit size and time interval of numerical analyses by DEM, Ref. [19]. Approaches to circumvent these limitations either depend on homogeneous material properties combined with hexagonal closest packing (hcp), the "unit cells" as introduced by Tavarez et al. [20], or imply a priori model restrictions by using a hybrid FEM/DEM model, e.g. [21].

As an ansatz to loosen this constraint for a class of DEM models, we propose a model order reduction method based on coherency in the dynamic behavior of particles. While initial flexibility of DEM is conserved, computational effort can be reduced significantly.

This paper is outlined as follows: At first, in Sects. 2 and 3 we present the basic concept of DEM and introduce the KLT in the formulation of model order reduction. Then, in Sect. 4 
we discuss our new approach for a hybrid DEM model. In Sect. 5 we present an example for the numerical simulation of a reduced order DEM model. Finally we summarize our findings.

\section{A simple DEM model}

Discrete element method models vary as much as the objects of analyses. However, there are a few common properties to characterize the DEM: structures that may represent bodies or fluids are not regarded as continua but as assemblies of discrete elements. As a result of this approach, forces act between discrete elements and are evaluated individually for each element. This results in a system of (formally) decoupled, more or less stiff equations of motion (5) and (6). There are two major branches of DEM that differ in the formulation of particle-particle forces: (1) Models that assign attracting and repulsing potential fields to each particle, merely used to model dynamics on atomistic scale ${ }^{1}$ and (2) Models that apply elasto-plastic contact laws, employing more complex neighborhood/contact detection algorithms, mainly used at macro-scale level.

In this paper, we focus on the procedural aspects of the DEM evaluation scheme itself. Therefore, we choose a simple DEM model: we apply spherical elements governed by potential forces. We represent specimen or particular regions of interest $\Omega$, with boundary $\partial \Omega$ by assemblies of particles $p_{i} \in \Sigma$, with coordinates $\left\{x_{i} y_{i} z_{i}\right\} \in \Omega \cup \partial \Omega$.

Let us use a cartesian inertial reference frame $K_{0}\left\{O, \mathbf{e}_{1}\right.$, $\left.\mathbf{e}_{2}, \mathbf{e}_{3}\right\}$ to describe the particle coordinates $\mathbf{x}_{i}:=\left[\begin{array}{ll}x_{i} & y_{i} \\ z_{i}\end{array}\right]^{T}$ and the angles of rotation $\boldsymbol{\varphi}_{i}:=\left[\phi_{i} \theta_{i} \psi_{i}\right]^{T}$ of particle $p_{i}$ about global axes $\mathbf{e}_{1}, \mathbf{e}_{2}$ and $\mathbf{e}_{3}$ at $\mathbf{x}_{i}$. Furthermore, we collect the coordinates of all particles by the vector of generalized coordinates

$\mathbf{q}:=\left[\mathbf{x}_{1} \mathbf{x}_{2} \ldots \mathbf{x}_{N} \varphi_{1} \varphi_{2} \ldots \varphi_{N}\right]^{T}$.

\subsection{Equation of motion}

If we agree that particles are subject to potential field forces, we can use Lagrange's method to derive the equations of motion. Let us express Lagrange's function by

$\mathcal{L}=T_{t}+T_{r}-V$,

with components

$$
T_{t}=\sum_{i=1}^{N} \frac{m_{i} \mathbf{x}_{i}^{T} \mathbf{x}_{i}}{2}
$$

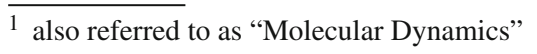

$T_{r}=\sum_{i=1}^{N} \frac{\omega_{i}^{T} \mathbf{I}_{i} \cdot \omega_{i}}{2}$

denoting the kinetic energy of $(\mathrm{t})$ ranslation and of (r)otation, and the potential energy $V$. Note, that the potential energy $V$ can be calculated by superposition of individual potentials $U_{i j}$ as

$V\left(\mathbf{x}_{1}, \ldots, \mathbf{x}_{N}, \boldsymbol{\varphi}_{1}, \ldots, \boldsymbol{\varphi}_{N}\right)=\sum_{i=1}^{N} \sum_{j=1, j \neq i}^{N} U_{i j}\left(d_{i j}\right)$,

where $d_{i j}:=\left\|\mathbf{x}_{i}-\mathbf{x}_{j}\right\|$ is the distance between particles $\mathrm{i}$ and $\mathrm{j}$. Then, from Lagrange's principle

$\frac{d}{d t} \frac{\partial \mathcal{L}}{\partial \dot{\mathbf{q}}_{i}}-\frac{\partial \mathcal{L}}{\partial \mathbf{q}_{i}}=0, \quad i=1,2, \ldots, N$

we directly obtain Newton's and Euler's equation for rigid bodies

$m_{i} \ddot{\mathbf{x}}_{i}=\sum_{j=1, j \neq i}^{N}-\frac{\partial U_{j}}{\partial \mathbf{x}_{i}}=\sum_{j=1, j \neq i}^{N} \mathbf{f}_{i j}$

$\mathbf{I}_{i} \cdot \dot{\boldsymbol{\omega}}_{i}+\boldsymbol{\omega}_{i} \times \mathbf{I}_{i} \cdot \boldsymbol{\omega}_{i}=\sum_{j=1, j \neq i}^{N}-\frac{\partial U_{j}}{\partial \boldsymbol{\varphi}_{i}}=\sum_{j=1, j \neq i}^{N} \boldsymbol{\ell}_{i j}$

where $i, j=1,2, \ldots, N$. This equation is the core of the DEM and appears to be quite simple. However, evaluating the individual particle-particle forces $\mathbf{f}_{i}$ and moments $\boldsymbol{\ell}_{i}$ is the actual challenge of the DEM algorithms and is numerically very expensive. Since we focus on spherical particles, we can disregard Eq. (6) and rewrite Eq. (5) in matrix representation

$\ddot{\mathbf{q}}=\mathbf{M}^{-1} \mathbf{f}$,

where $\mathbf{M}$ is the diagonal mass matrix and $\mathbf{f}$ is the vector of resultant particle forces.

\subsection{Particle forces}

Neglecting contact problems, we are left with potential driven particle-particle interactions. The most popular potentials in the framework of DEM are Gravitational potential of two masses, Coulomb potential as a result of electric charge, Harmonic potential as in Hooke's law, van der Waals potential and the more complex Lennard-Jones potential, ref. e.g. [22].

The Lennard-Jones potential combines an attractive and a repulsive part, where the repulsive portion is similar to van der Waals potential

$U_{i j}\left(d_{i j}\right)=\alpha \varepsilon\left(\left(\frac{\sigma}{d_{i j}}\right)^{n}-\left(\frac{\sigma}{d_{i j}}\right)^{m}\right), \quad m<n$ 


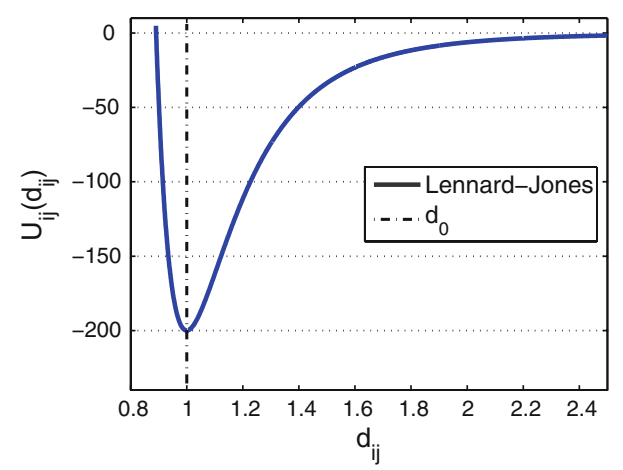

Fig. 1 Lennard-Jones potential $U_{i j}$ versus particle distance

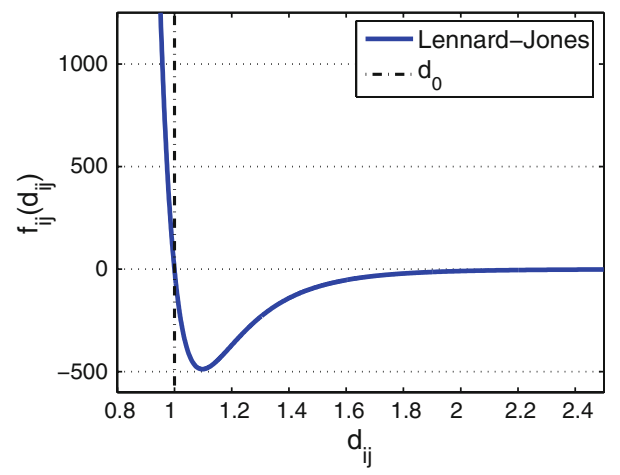

Fig. 2 Lennard-Jones force $f_{i j}$ versus particle distance

where $\alpha=\frac{1}{n-m}\left(\frac{n^{n}}{m^{m}}\right)^{\frac{1}{n-m}}$ and parameter $\varepsilon$ describes the magnitude of the potential, while $\sigma$ determines the neutral distance such that $d_{0}:=\left.\frac{d U_{i j}}{d d_{i j}}\right|_{d_{0}}=0$.

To demonstrate that our model order reduction procedure holds under extreme nonlinear governing equations, we chose Lennard-Jones potential for particle-particle forces

$\mathbf{f}_{i j}\left(d_{i j}\right)=24 \varepsilon \frac{1}{d_{i j}^{2}}\left(\frac{\sigma}{d_{i j}}\right)^{6}\left(1-2\left(\frac{\sigma}{d_{i j}}\right)^{6}\right) \mathbf{d}_{i j}$,

with the choice of $m=6, n=12$ leading to $d_{0}=2^{1 / 6} \cdot \sigma$ and $\varepsilon=200 .^{2}$ Figures 1 and 2 display potential and force vs. particle-particle distance $d_{i j}$, negative force values act attractive, while positive values do the opposite. Since both curves, potential and force, rapidly decay to zero as the distance increases we can regard Lennard-Jones potential as a short range potential. Provided our above mentioned choice of parameters, the Lennard-Jones force has a range of about 2.5 times the neutral distance. Thus, we can define a "cut-off distance" $d_{\text {cut }}=2.5 \cdot d_{0}$ and we can limit the number

\footnotetext{
${ }^{2}$ A more general approach to potential fields and interacting particle dynamics can be found in [24].
}

of considered particle-particle forces in the resultant force

$\mathbf{f}_{i} \approx \sum_{j=1}^{C<N} \mathbf{f}_{i j}, \quad j(i) \in\left\{j \mid \quad\left\|\mathbf{x}_{j}-\mathbf{x}_{i}\right\| \leq r_{\mathrm{cut}}\right\}$,

to $C$ that is significantly smaller that the number of total particles $N$.

\subsection{Boundary conditions}

It is quite common to apply periodic boundary conditions when modeling systems with potential particle forces. This is done by folding the configuration space in either direction and bring them to connection. In order to model rigid boundaries and driving forces in addition to the "free particles" $p_{i}^{\Theta} \in \Theta \subset \Sigma$, "constraint particles" $p_{i}^{\Phi} \in \Phi \subset \Sigma$ are introduced that bear the same potential as the free particles, but are subject to holonomic constraints $\phi\left(\mathbf{x}_{1}, \mathbf{x}_{2}, \ldots, \mathbf{x}_{N}, t\right)=0$.

\subsection{Neighborhood lists}

Taking into account the limited range of potentials $U_{i}$ and the fact that $\mathbf{f}_{i j}=\mathbf{f}_{j i}$, DEM routines evaluate a fraction of all particle-particle forces, only. The calculation of forces is guided by an array of "neighborhood lists" $L_{i}$, that for each particle $p_{i}$ lists its "left sided" neighbors. These particles $p_{j} \in L_{i}$ are chosen by

$$
\begin{aligned}
p_{j} \in L_{i}:= & \left\{p_{j} \mid \quad\left\|\mathbf{x}_{j}-\mathbf{x}_{i}\right\|<d_{\text {cut }}+d_{\text {res }}\right. \\
& \wedge\left(x_{j}<x_{i}\right) \wedge\left(y_{j}<y_{i}\right) \\
& \forall j=1,2, \ldots, N, j \neq i\},
\end{aligned}
$$

to prevent redundant calculations. Additional distance $d_{\text {res }}$ is a reserve distance that reduced the number of necessary list updates by considering particle inertia.

However, despite these simplifications the evaluation of the right hand side of (5) still involves a large number of numerical operations for each time step, thus the need for a further model reduction persists.

\subsection{Numerical procedure}

Although not obvious, due to (9) and (10) the equations of motion (5) turn out to be rather stiff. Thus, we use a fourth order multi-value implicit integration scheme in Gear's predictor-corrector notation, as proposed in [23]. The numerical integration routine is executed in the main loop: Predictor $\mathcal{P} \rightarrow$ Error evaluation $\mathcal{E} \rightarrow$ Corrector $\mathcal{C}$, as per $\mathcal{P}(\mathcal{E C})^{m}$.

For first order differential system representation, the set of derivatives for each particle $p_{i}$ is combined in

$\mathbf{y}_{i}(n)=\left[\mathbf{x}_{i}(n) \mathbf{x}_{i}^{\prime}(n) \mathbf{x}_{i}^{\prime \prime}(n) \mathbf{x}_{i}^{\prime \prime \prime}(n) \mathbf{x}_{i}^{\prime \prime \prime \prime}(n)\right]^{T}$, 
where we used $\mathbf{x}^{\prime}(n):=d(\mathbf{x}(n)) / d n$ as abbreviation. For the sake of clarity, in this section we neglect vector notation of the particle position, write time in form of $x_{n}^{\prime}:=x^{\prime}(n)$ and omit particle number index " $i$ ". The integration routine of the simple DEM model then is evaluated by

Predictor step $\mathcal{P}$; we simply extrapolate the current particle position from its current state

$\mathbf{y}_{p}=\left[\begin{array}{r}x_{n}+x_{n}^{\prime} h+\frac{1}{2} x_{n}^{\prime \prime} h^{2}+\frac{1}{6} x_{n}^{\prime \prime \prime} h^{3}+\frac{1}{24} x_{n}^{\prime \prime \prime \prime} h^{4} \\ x_{n}^{\prime}+x_{n}^{\prime \prime} h+\frac{1}{2} x_{n}^{\prime \prime \prime} h^{2}+\frac{1}{6} x_{n}^{\prime \prime \prime \prime} h^{3} \\ x_{n}^{\prime \prime}+x_{n}^{\prime \prime \prime} h+\frac{1}{2} x_{n}^{\prime \prime \prime \prime} h^{2} \\ x_{n}^{\prime \prime \prime}+x_{n}^{\prime \prime \prime \prime} h\end{array}\right]$.

Error evaluation $\mathcal{E}$; requiring the differential equation to satisfy $\mathbf{y}_{n+1}^{\prime}=\mathcal{F}\left(\mathbf{y}_{n+1}\right)$, which we find to equal

$x_{p}^{\prime \prime}=\frac{1}{m} f\left(x_{p}\right)$.

Thus, we can determine the extrapolation error by

$\epsilon=\frac{1}{m} f\left(x_{p}\right)-x_{p}^{\prime \prime}$.

Corrector $\mathcal{C}$; the corrected estimate then becomes

$\mathbf{y}_{n+1}=\left[\begin{array}{c}x_{p}+c_{0} \epsilon h^{2} \\ x_{p}^{\prime}+c_{1} \epsilon \frac{h}{2} \\ f\left(x_{p}\right) \\ x_{p}^{\prime \prime \prime}+c_{3} \epsilon \frac{1}{3 h} \\ x_{p}^{\prime \prime \prime \prime}+c_{4} \epsilon \frac{12}{h^{2}}\end{array}\right]$

with corrector coefficients $c_{0}, c_{1}, c_{3}$ and $c_{4}$.

\section{Basics of KLT}

We employ the Karhunen-Loève transform (KLT) in a Ritz-ansatz to change the representation of the generalized coordinates $\mathbf{q}$, based on the reference frame $K_{0}\left\{O, \mathbf{e}_{1}, \mathbf{e}_{2}\right.$, $\left.\ldots, \mathbf{e}_{N}\right\}$ to a representation $\boldsymbol{\alpha}$ based on KLT-basis $\Psi=$ $\left\{\boldsymbol{\psi}_{1}, \boldsymbol{\psi}_{2}, \ldots, \boldsymbol{\psi}_{N}\right\}$. Unless the coordinates $\mathbf{q}$ are uncorrelated $\boldsymbol{\alpha}$ by far is the more efficient representation of the particle motion. ${ }^{3}$

Figure 3 gives an insight into the idea of KLT for a two-dimensional configuration space: Let the ellipse resemble data of dynamic system behavior of $\mathbf{q}(t)$. When positions are represented by tupel $\left\{y_{1}, y_{2}\right\}$ according to reference frame $K_{0}\left\{O, \mathbf{e}_{1}, \mathbf{e}_{2}\right\}$, initially, it takes both coordinates for a reasonable good description. Since coordinates $y_{1}$ and $y_{2}$ are correlated, statistically, we can find a reference frame

\footnotetext{
3 Various authors e.g. Meyer and Matthies [25] or Lall et al. [26] have shown that the KLT-basis is the optimum basis with respect to least squares measurement.
}

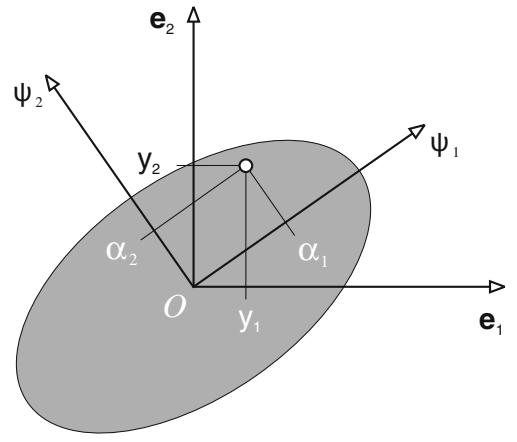

Fig. 3 Coordinate transform by KLT

$K_{0}\left\{O, \psi_{1}, \psi_{2}\right\}$, that better fits the overall system behavior. Thus, provided that the ellipse is flat enough, we may represent the system motion by just the first coordinate $\alpha_{1}$ without significant loss of information, e.g. [27]. In this section we briefly introduce the mathematical background of the KLT procedure and refer to e.g. [28] for a detailed discussion.

The KLT is a signal-dependent transform which means that basis $\Psi$ depends on the process under investigation: $\mathbf{q}(t)$. With discretized time $t=n \cdot h$ and discrete particles the KLTexpansion of particle coordinates can be expressed by

$\tilde{\mathbf{q}}(n)=\mathbf{q}(n)-\overline{\mathbf{q}}=\mathcal{F}(n)=\sum_{i=1}^{N} \alpha_{i}(n) \cdot \boldsymbol{\psi}_{i}$,

as superposed products of time-dependent coefficients $\alpha_{i}(n)$ and "characteristic functions" $\psi_{i}$, while mean-value vector $\overline{\mathbf{q}}$. Equation (17) equals

$\tilde{\mathbf{q}}(n)=\boldsymbol{\Psi} \boldsymbol{\alpha}(n)$

and the inverse transform

$\boldsymbol{\alpha}(n)=\boldsymbol{\Psi}^{-1} \tilde{\mathbf{q}}(n)$.

in vector/matrix notation, with $\boldsymbol{\alpha}(n):=\left[\alpha_{1}(n) \alpha_{2}(n) \ldots\right.$ $\left.\alpha_{n}(n)\right]^{T}$ and transformation matrix $\boldsymbol{\Psi}:=\left[\boldsymbol{\psi}_{1} \boldsymbol{\psi}_{2} \cdots \boldsymbol{\psi}_{N}\right]$. Following Karhunen [29], we can find characteristic functions that incorporate coherent motion in a set of given data of $\mathcal{D}=\{\mathbf{q}(1), \mathbf{q}(2), \ldots, \mathbf{q}(n)\}$ if we require uncorrelated coordinates

$E\left\{\alpha_{i}(n) \alpha_{j}(n)\right\}=\lambda_{j} \delta_{i j}, \quad i, j=1, \ldots, n$,

where $\lambda_{j} \in \mathbb{R} \geq 0$ are unknown scalars and $\delta_{i j}$ is Kronecker's function. We are free to demand characteristic functions $\boldsymbol{\psi}_{i}$ to be orthogonal. Thus, assuming normalized characteristic functions relation $\boldsymbol{\Psi}^{-1}=\boldsymbol{\Psi}^{T}$ holds for the KLT-transform matrix. Inserting Eq. (17) into Eq. (20) and considering orthonormal $\psi_{i}$ leads to

$E\left\{\boldsymbol{\psi}_{i}^{T} \mathbf{q q}^{T} \boldsymbol{\psi}_{j}\right\}=\lambda_{j} \delta_{i j}, \quad i, j=1,2, \ldots, N$.

We can extract time-independent $\boldsymbol{\psi}_{i, j}$ from estimate $E\{\cdot, \cdot\}$ and regard $\mathbf{q}(n)$ as vector random process. Thus, Eq. (21) turns into 
$\boldsymbol{\psi}_{i}^{T} \mathbf{C}_{q q} \boldsymbol{\psi}_{j}=\lambda_{j} \delta_{i j}, \quad i, j=1,2, \ldots, N$.

that is satisfied by solutions $\psi_{i}, i=1,2, \ldots, N$ of eigenvalue problem

$\mathbf{C}_{q q} \boldsymbol{\psi}_{i}=\lambda_{i} \boldsymbol{\psi}_{i}, \quad i=1,2, \ldots, N$,

with covariance matrix $\mathbf{C}_{q q}=E\left\{\mathbf{q q} \mathbf{q}^{T}\right\}$ of $\mathbf{q}(n)$.

Equation (23) gives $N$ eigenvalues $\lambda_{i}$ and $N$ eigenvectors $\psi_{i}$, that can be normalized by Gram-Schmidt procedure. Basis $\Psi$ spans vector space $\mathcal{Q} \subset \mathbb{R}^{N}$ of system dynamics $\mathbf{q}$ completely. The KLT is a unique coordinate transform. Provided that all characteristic functions are considered KLT is a lossless transform, as well.

In general, the motion represented by the few first weighing factors $\alpha_{i}, i=1,2, \ldots, M \ll N$ (ordered by the eigenvalues $\lambda_{i}$ ) along the corresponding characteristic functions $\psi_{i}$ cover more than $95 \%$ of the power/kinetic energy of the system. Therefore, truncating the sum of characteristic functions at $i=M$ in Eq. (17) we can approximate $N$-dimensional system of equations of motion

$\ddot{\mathbf{q}}=\mathcal{F}(\mathbf{q}, \dot{\mathbf{q}}, n)$

in coordinates of $\mathbf{q}$ by reduced $M$-dimensional system of equations

$\ddot{\mathbf{q}} \approx \tilde{\mathbf{\Psi}}^{T} \ddot{\tilde{\alpha}}+\overline{\mathbf{q}}$,

where

$\ddot{\tilde{\boldsymbol{\alpha}}}=\mathcal{F}(\tilde{\boldsymbol{\alpha}}, \dot{\tilde{\boldsymbol{\alpha}}}, n)$

and reduced transform matrix $\tilde{\Psi}$.

Let us note that $\mathcal{D}$ is collected by the "method of snapshots" as introduced by Sirovich [30] from representative simulation data. Due to fixed $\mathcal{D}$ approximation quality of Eq. (25) might change for certain perturbed systems. In their recent publication Glösmann and Kreuzer [31] address this issue and discuss a measure to monitor the performance of reduced KLT-basis $\tilde{\boldsymbol{\Psi}}$.

\section{A hybrid DEM-KLT model}

In various problems, analyzed by DEM, e.g. impact or indentation of solids, the actual region of high stress (potential energy $V$ ) and/or high velocity (kinetic energy $T$ ), respectively, is limited. Consequently, there is no need to model the entire specimen/volume (entity $\Omega \cup \partial \Omega$ ) in all detail. Thus, allowing for some (controlled) simulation error, we can separate discretized entity $\Sigma \subset \Omega \cup \partial \Omega$ into regions of high interest, that are modeled by free particles $p_{i}^{\Theta} \in \Theta$ and regions of little interest, represented by groups of "cluster particles" $p_{k i}^{\Gamma} \in \Gamma_{k}$, where $\Theta \subset \Sigma, \Gamma_{k} \subset \Sigma$ and $\Theta \cap \Gamma_{k}=\emptyset$ while $\Gamma_{k} \cap \Gamma_{j}=\emptyset \forall k \neq j$.

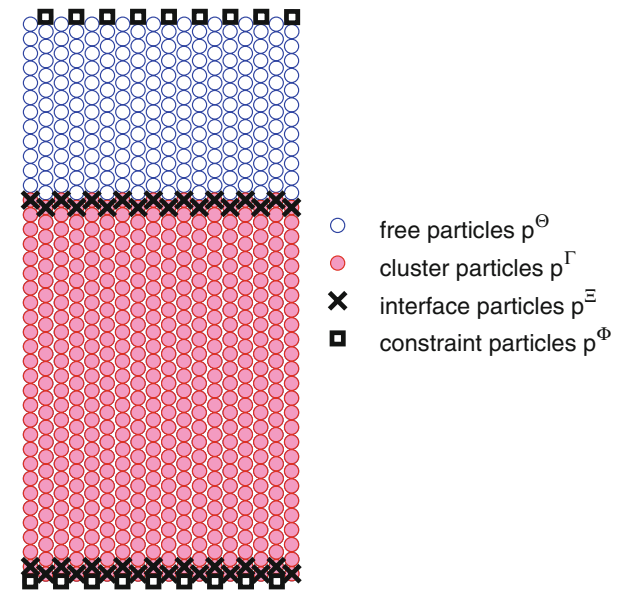

Fig. 4 Cantilever beam modeled by hybrid DEM/KLT method particle types

If we find coherency in the dynamics of all particles $p_{k i}^{\Gamma}$ of cluster $k$, we can represent their behavior by characteristic functions $\boldsymbol{\psi}_{k i}$. Thus, we can express the motion of all cluster particles $p_{k i}^{\Gamma}$ of cluster $k$ by a set of reduced order differential equations of type (26). ${ }^{4}$

This ansatz leads to a reduced order hybrid DEM/KLTmodel, where the reduction is well controlled by the choice of the portion of cluster particles out of the total number of particles. The stronger the motion of the particles per cluster is coupled, the less equations (degrees of freedom) per cluster are necessary to describe its dynamics. Thus, locally a decisive order reduction is achieved.

At this point, we have to formulate the interface equations for displacements/forces between $\Theta$ and $\Gamma_{k}$. This is an easy task to do and the solution is quite intuitive: throughout the model reduction, we never change the basic model itself. We still model entity $\Omega \cup \partial \Omega$ by discrete elements. However, we partially project the coordinates $\mathbf{q}_{k}$ of the cluster particles $p_{k i}^{\Gamma}$ onto the reduced KLT-basis of coherent particle motion $\Psi^{k}$ for representation. Therefore, in order to couple cluster particles and free particles, we have to identify those cluster particles, that have an effect on the surrounding free particles. These we call "interface particles" $p_{k i}^{\Xi} \in \Xi_{k} \subset \Gamma_{k}$. Figure 4 displays the different particle types, exemplarily, at the hybrid DEM/KLT model of a cantilever beam that is suspended at the bottom and driven at the top.

The reduced order hybrid DEM/KLT method involves the following steps

1. Simulation of full order DEM-model; to obtain a reference data base $\mathcal{D}$,

2. Identification of clusters $\Gamma_{k} /$ cluster particles $p_{k i}^{\Gamma}$; regions of little interest, subject to model order reduction,

\footnotetext{
${ }^{4}$ Since we apply the KLT to cluster particles only, we omit superscript " $\Gamma$ " in vector of coordinates $\alpha$ and characteristic functions $\psi$.
} 
3. Identification of interface particles $p_{k i}^{\Xi}$ for each cluster $k$,

4. Calculation of characteristic functions $\boldsymbol{\psi}_{k i}$; KLTprocedure for all particles $p_{k i}^{\Gamma}$ of each cluster $k$,

5. Model order reduction; setting up the hybrid equations of motion of free particles $p_{i}^{\Theta}$ with reduced order equations and of cluster particles $p_{k i}^{\Gamma}$ of each cluster and

6. Approximation of macroscopic Lennard-Jones forces; approximation of stiffness parameters $\mathbf{k}_{k}$ for each cluster $k$,

that are discussed shortly in the following.

\subsection{Reference data base}

The full size DEM-model simulation provides necessary data base $\mathcal{D}$ to identify the regions of little interest $\Gamma_{k}$ and to calculate the covariance matrices $\mathbf{C}_{q q k}$ for each cluster $k$.

\subsection{Identifying cluster particles}

Depending on the criterion, potential energy, kinetic energy or a combination of both, the cluster particles can be identified by their contribution to the total energy of the system, using

$$
\begin{aligned}
& p_{i}^{\Gamma}=\left\{p_{i} \mid \bar{V}\left(p_{i}\right) \leq \mu \cdot \frac{1}{N} \sum_{j=1}^{N} \bar{V}\left(p_{j}\right) \forall p_{i}, p_{j} \in \Sigma\right\} \\
& p_{i}^{\Gamma}=\left\{p_{i} \mid \bar{T}\left(p_{i}\right) \leq \mu \cdot \frac{1}{N} \sum_{j=1}^{N} \bar{T}\left(p_{j}\right) \forall p_{i}, p_{j} \in \Sigma\right\} \text { or } \\
& p_{i}^{\Gamma}=\left\{p_{i} \mid\left(\bar{T}\left(p_{i}\right)+\bar{V}\left(p_{i}\right)\right) \leq \mu \cdot \frac{1}{N} \sum_{j=1}^{N}\left(\bar{T}\left(p_{j}\right)+\bar{V}\left(p_{j}\right)\right)\right. \\
&\left.\forall p_{i}, p_{j} \in \Sigma\right\}
\end{aligned}
$$

respectively, where $\mu$ is a predefined constant factor, e.g. $\mu=10 \% .^{5}$

\subsection{Identifying interface particles}

The interface particles $p_{k i}^{\Xi} \in \Xi_{k} \subset \Gamma_{k}$ can be separated from the cluster particles by the entries of their neighborhood lists $L_{i}$. While the particles $p_{k i}^{\Gamma}$ of one cluster $k$ only refer to and are referred to by particles of the same cluster, the interface particle also are linked to particles outside of cluster $k$.

\footnotetext{
${ }^{5}$ Here, symbol ${ }^{-}$resembles the average of $\cdot$ in time.
}

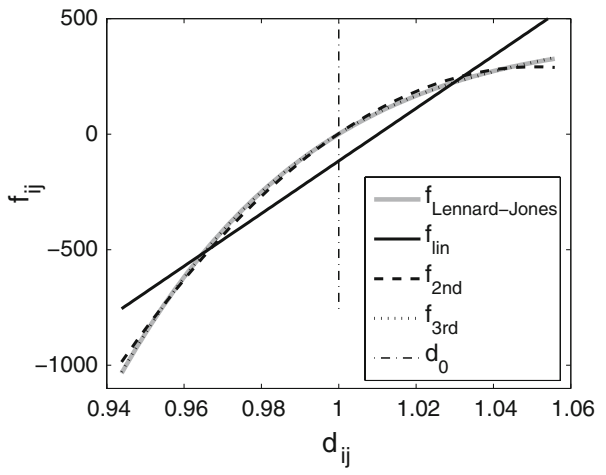

Fig. 5 Approximations of Lennard-Jones force

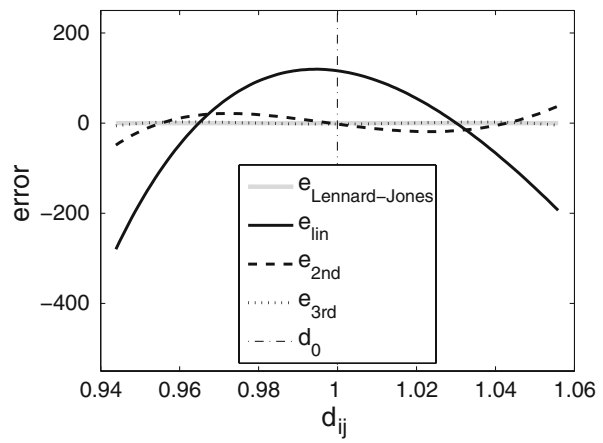

Fig. 6 Approximation error

Fig. 7 Sketch of suspended cantilever beam

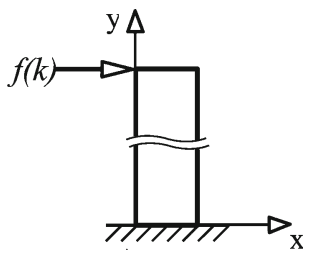

\subsection{Characteristic functions}

The dynamic behavior of particles $p_{k i}^{\Gamma}$ of each cluster $k$ can be described by one set of equations (26), using reduced basis $\Psi_{k}$. Each set of characteristic functions $\left\{\boldsymbol{\psi}_{k 1}, \boldsymbol{\psi}_{k 2}, \ldots, \boldsymbol{\psi}_{k M}\right\}$ is calculated by Eq. (23), while covariance matrix $\mathbf{C}_{q q k}$ is derived from the reference data base $\mathcal{D}$.

\subsection{Reduced order hybrid DEM/KLT equation of motion}

Since we partition the entity of particles $\Sigma$ of a DEM model into free particles $\Theta$ and groups of cluster particles $\Gamma$ (with bounding interface particles), we also have to divide coordinate vector $\mathbf{q}=\left[\mathbf{q}_{\Theta} \mathbf{q}_{\Gamma}\right]^{T}$ with $\mathbf{q}_{\Gamma}=\left[\mathbf{q}_{1} \mathbf{q}_{2} \cdots \mathbf{q}_{Z}\right]^{T}$, mass matrix $\mathbf{M}=\operatorname{diag}\left\{\mathbf{M}_{\Psi} \mathbf{M}_{1} \mathbf{M}_{2} \cdots \mathbf{M}_{Z}\right\}$ and vector of generalized forces $\mathbf{f}=\left[\mathbf{f}_{\Theta}+\mathbf{f}_{\Xi}+\mathbf{f}_{\Psi}\right]$ of Eq. (7), accordingly.

In addition, for each cluster $k$, we replace $\mathbf{q}_{k}$ by Weighing Factors $\boldsymbol{\alpha}_{k}$, Eq. (17), using the most significant characteristic functions $\tilde{\boldsymbol{\Psi}}_{k}=\left[\boldsymbol{\psi}_{k 1} \boldsymbol{\psi}_{k 2} \ldots \boldsymbol{\psi}_{k M}\right]$, only. Thus, we obtain the reduced order hybrid DEM/KLT equation of motion 
Table 1 Sensitivity of reduced order hybrid DEM/KLT model $\tilde{\boldsymbol{\Psi}}$ simulation results against different types of excitation compared to full size DEM model simulation results

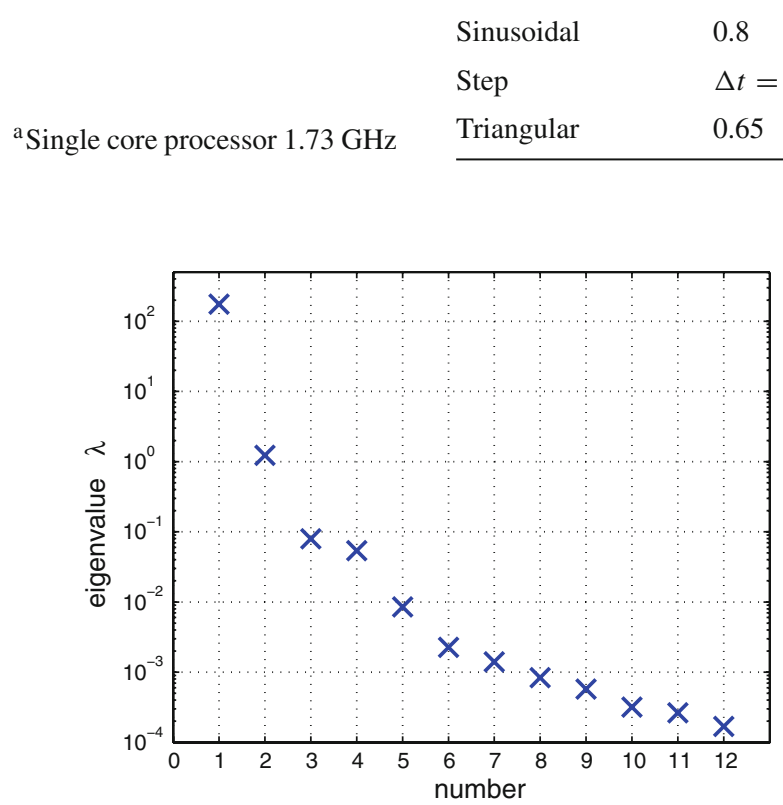

Fig. 8 First eight eigenvalues of cluster motion (logarithmic scale)

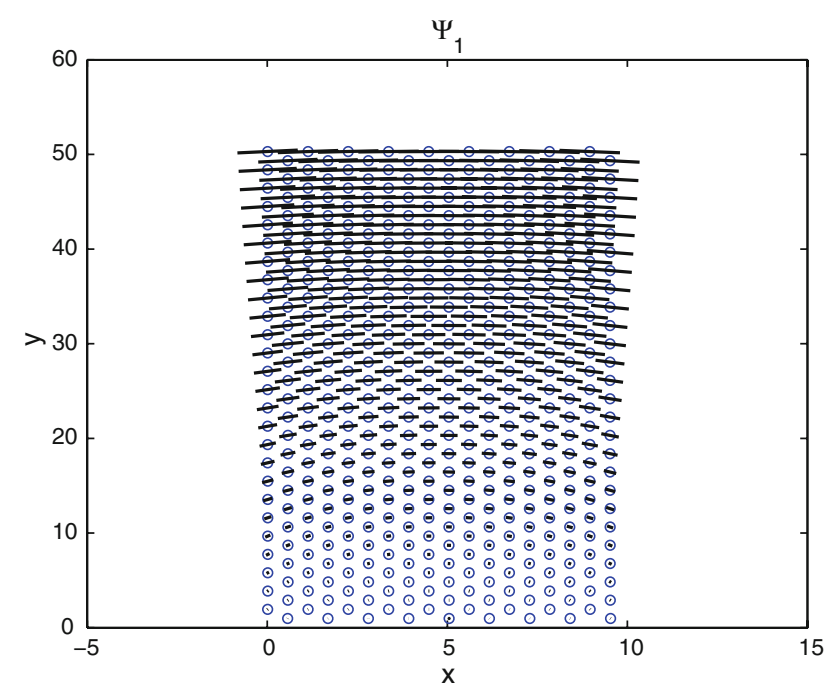

Fig. 9 Characteristic function $\psi_{1}$

$$
\begin{aligned}
& \ddot{\mathbf{q}} \approx \mathbf{M}_{\Theta}^{-1}\left[\mathbf{f}_{\Theta}+\mathbf{f}_{\Xi}+\mathbf{f}_{\Phi}\right] \\
& \ddot{\tilde{\boldsymbol{\alpha}}}_{1}=\tilde{\boldsymbol{\Psi}}_{1}^{T} \mathbf{M}_{1}^{-1}\left[\mathbf{k}_{1}\left(\tilde{\boldsymbol{\Psi}}_{1} \tilde{\boldsymbol{\alpha}}_{1}\right)+\mathbf{f}_{\Xi 1}\right] \\
& \ddot{\tilde{\boldsymbol{\alpha}}}_{2}=\tilde{\boldsymbol{\Psi}}_{2}^{T} \mathbf{M}_{2}^{-1}\left[\mathbf{k}_{2}\left(\tilde{\boldsymbol{\Psi}}_{2} \tilde{\boldsymbol{\alpha}}_{2}\right)+\mathbf{f}_{\Xi 2}\right] \\
& \vdots \\
& \ddot{\tilde{\boldsymbol{\alpha}}}_{Z}=\tilde{\boldsymbol{\Psi}}_{Z}^{T} \mathbf{M}_{Z}^{-1}\left[\mathbf{k}_{Z}\left(\tilde{\boldsymbol{\Psi}}_{Z} \tilde{\boldsymbol{\alpha}}_{Z}\right)+\mathbf{f}_{\Xi Z}\right],
\end{aligned}
$$

where $\mathbf{f}_{\Theta}$ describes forces between free particles $p^{\Theta}, \mathbf{f}_{\Xi}$ describes forces acting between free particles $p^{\Theta}$ and interface particles $p^{\Xi}$, while $\mathbf{f}_{\Phi}$ resembles driving forces acting between constraint particles $p^{\phi}$ and free particles $p^{\Theta} /$ interface particles $p^{\Xi}$, respectively. Vectors $\mathbf{k}_{k}$ describe the nonlinear macroscopic stiffness of cluster $k$. Vectors $\mathbf{f}_{\Xi k}$ contain the coordinates of force vector $\mathbf{f}_{\Xi}$ acting on the cluster particles of clusters $k$.

This system of equations can be simplified further, if we find that matrices $\mathbf{K}_{k}$ of linearized $\mathbf{k}_{k}$ sufficiently approximate the macroscopic stiffness of cluster $k$. Then, we can rewrite Eq. (29) by

$$
\begin{aligned}
& \ddot{\mathbf{q}} \approx \mathbf{M}_{\Theta}^{-1}\left[\mathbf{f}_{\Theta}+\mathbf{f}_{\Xi}+\mathbf{f}_{\Phi}\right] \\
& \ddot{\tilde{\boldsymbol{\alpha}}}_{1}=\tilde{\boldsymbol{\Psi}}_{1}^{T} \mathbf{M}_{1}^{-1}\left[\mathbf{K}_{1} \tilde{\boldsymbol{\Psi}}_{1} \boldsymbol{\alpha}_{1}+\mathbf{f}_{\Xi 1}\right] \\
& \ddot{\tilde{\boldsymbol{\alpha}}}_{2}=\tilde{\boldsymbol{\Psi}}_{2}^{T} \mathbf{M}_{2}^{-1}\left[\mathbf{K}_{2} \tilde{\boldsymbol{\Psi}}_{2} \boldsymbol{\alpha}_{2}+\mathbf{f}_{\Xi 2}\right] \\
& \vdots \\
& \ddot{\tilde{\boldsymbol{\alpha}}}_{Z}=\tilde{\boldsymbol{\Psi}}_{Z}^{T} \mathbf{M}_{Z}^{-1}\left[\mathbf{K}_{Z} \tilde{\boldsymbol{\Psi}}_{Z} \boldsymbol{\alpha}_{Z}+\mathbf{f}_{\Xi Z}\right]
\end{aligned}
$$

that can be integrated very efficiently.

\subsection{Approximation of macroscopic Lennard-Jones forces}

There are two important requirements for the DEM/KLT method to significantly reduce the numerical integration effort: Firstly, the KLT procedure is used to identify the most significant characteristic functions (eigenforms) of cluster motion and thus to reduced the degrees of freedom. Secondly and equally important, it is necessary to approximate the macroscopic cluster stiffnesses by $\mathbf{k}_{k}$ or even $\mathbf{K}_{k}$, respectively.

Macroscopic stiffness vector/stiffness matrix of each cluster $k$ can be derived from the reference data $\mathcal{D}$ by e.g. least squares procedure. Figures 5 and 6 show least squares approximation and error of Lennard-Jones particle forces by polynomials up to third order. We can observe, that although Eq. (9) is of 12th order, already polynomials of third order can be used to approximate Lennard-Jones forces quite good. 


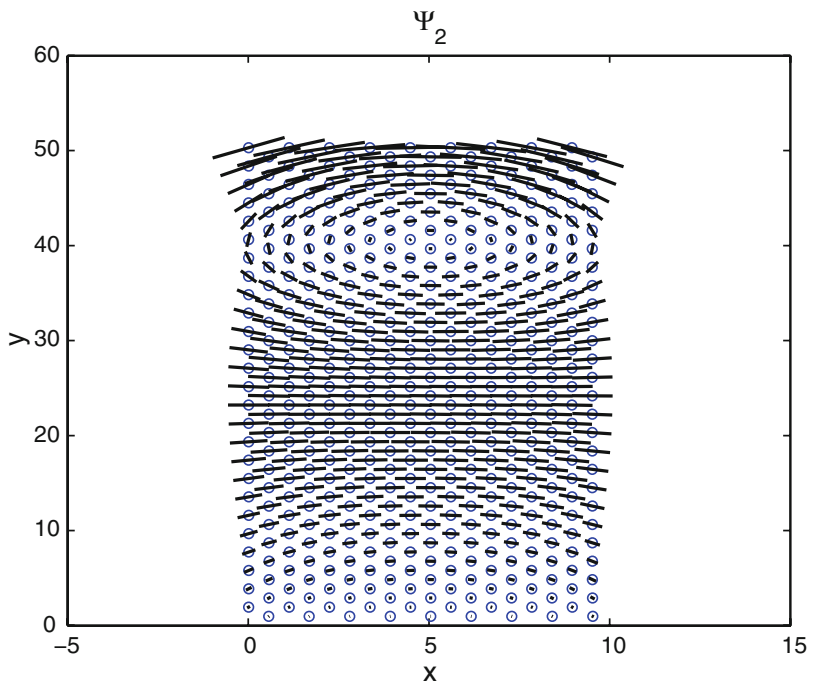

Fig. 10 Characteristic function $\psi_{2}$

\section{An exemplary study: dynamics of a cantilever beam}

We study the dynamic behavior of a cantilever beam, exemplarily. The cantilever beam is driven at its top end by transverse sinusoidal excitation. Fig. 7 shows a sketch of the set up.

We follow the hybrid DEM/KLT-method as proposed in Sect. 4: at first we simulate the motion of the 2D-DEM model for one period and store positions, velocities and resultant forces of particles in a data set $\mathcal{D}$. Then, we identify the particles of minor interest. We choose cluster particles $p_{i}^{\Gamma}$ to comply with kinetic energy criterion of Eq. (27), where $\mu=1.5$. Finally, we simulate the hybrid DEM/KLT model and compare the results.

With initial conditions $\left\{\mathbf{q}(0)=\mathbf{q}_{0}, \dot{\mathbf{q}}(0)=\mathbf{0}\right\}$ and a sinusoidal excitation ( $f=0.2 \mathrm{~Hz}, a=3 d_{0}$, confer Table 1) below the first eigenmode, the DEM model of the cantilever beam shows the typical motion pattern. However, the DEM model is capable of revealing the transient dynamics as the transversal displacement wave travels from top to bottom, as well.

\subsection{Identified cluster particles}

We use simulation data set $\mathcal{D}$ to select the cluster particles and find that the $p_{i}^{\Gamma}$ are located in a well defined area of $\Omega$. Moreover, the cluster particles form an interconnecting, closed set (group without inclusions), as shown in Fig. 4. While the total number of particles is 702 , the number of identified cluster particles is 468 . Thus, we will keep 234 free particles $p_{i}^{\Theta}$.

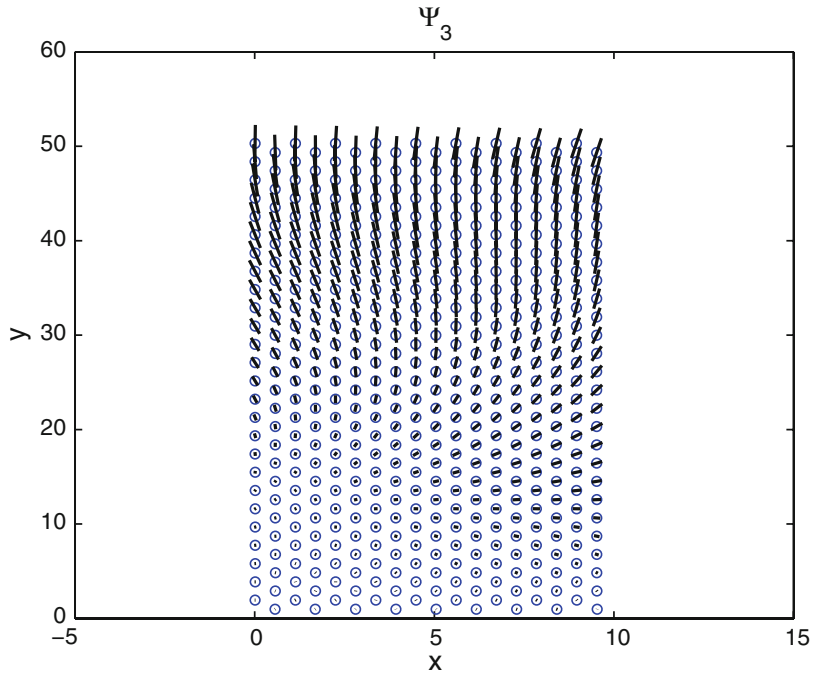

Fig. 11 Characteristic function $\psi_{3}$

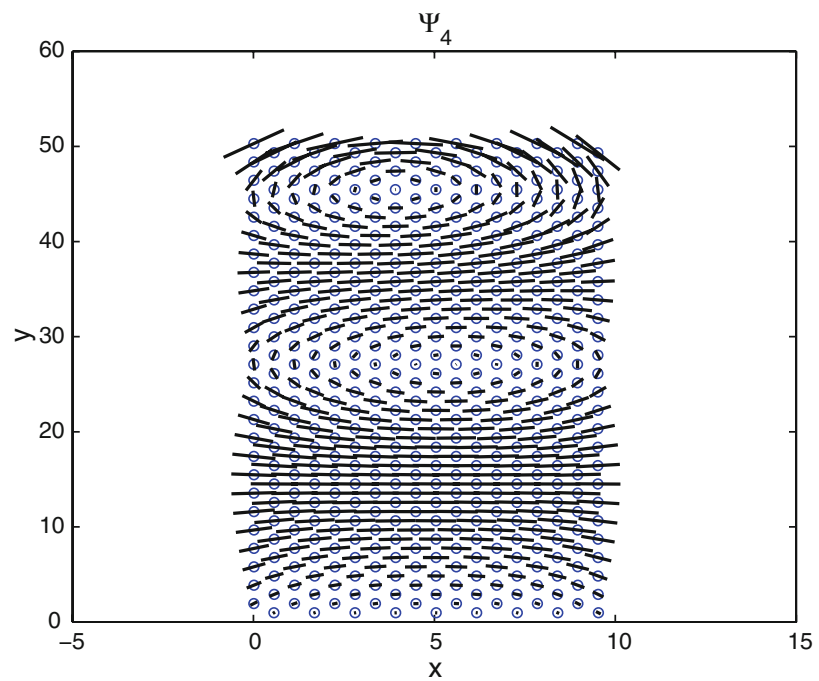

Fig. 12 Characteristic function $\psi_{4}$

\subsection{Karhunen-Loève decomposition}

The KLT decomposition of the displacements of the cluster particles gives 468 eigenvalues $\lambda_{j}$ and corresponding characteristic functions $\psi_{j}$. The first 12 eigenvalues are displayed in Fig. 8. A logarithmic scale ${ }^{6}$ is necessary to differentiate between eigenvalues number 4 and higher. Since eigenvalue $\lambda_{j}$ represents the contribution of the corresponding characteristic function $\psi_{j}$ to the system dynamics, it is obvious that only a small number of characteristic functions is necessary to capture the relevant cluster motion.

The characteristic functions $\boldsymbol{\psi}_{j}$ represent 2 dimensional empirical eigenforms of all cluster particles. Figures 9, 10,

\footnotetext{
6 The change in magnitude of the first two eigenvalues even exceeds an exponential decay.
} 


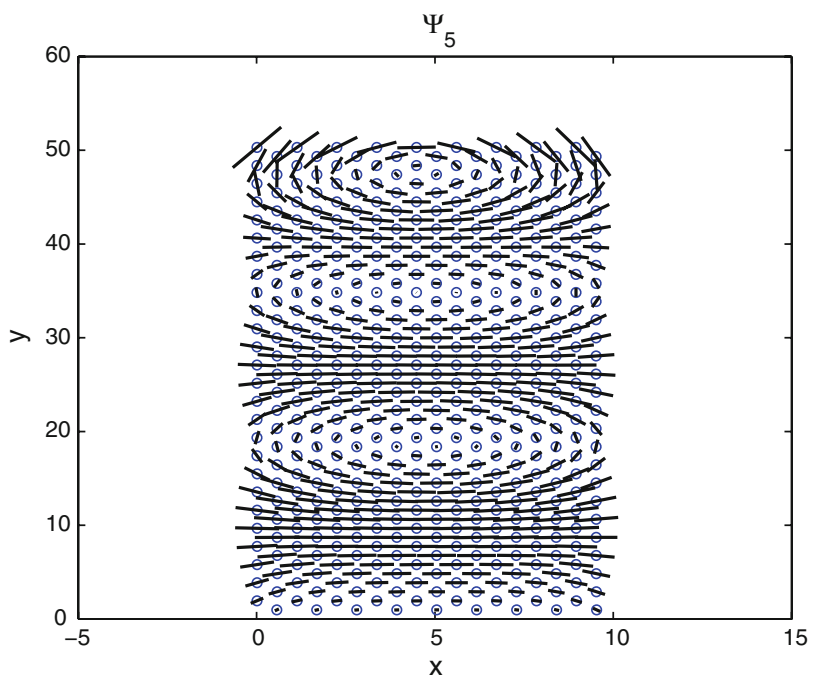

Fig. 13 Characteristic function $\psi_{5}$

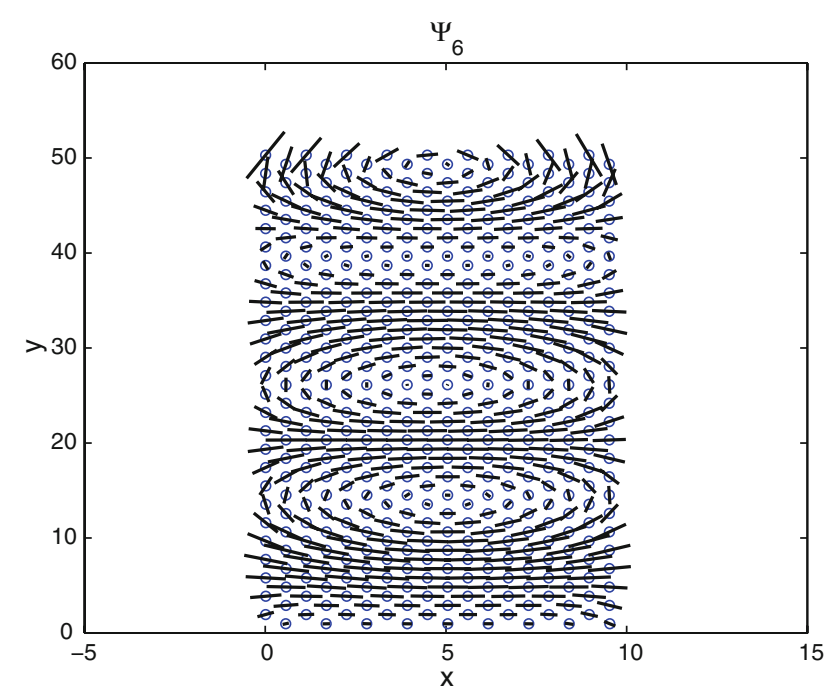

Fig. 14 Characteristic function $\psi_{6}$

$11,12,13,14,15,16,17,18,19$ and 20 show the 12 most important characteristic functions corresponding to the first 12 eigenvalues of $\mathrm{KL}$ decomposition of data $\mathcal{D}$. The circles represent individual particles, while the lines are gradients of particle motion. ${ }^{7}$ Thus, nodes and anti-nodes of vibration modes can well be detected. Characteristic functions $\boldsymbol{\psi}_{1}, \boldsymbol{\psi}_{2}$, $\boldsymbol{\psi}_{4}, \boldsymbol{\psi}_{5}, \boldsymbol{\psi}_{6}, \boldsymbol{\psi}_{8}, \boldsymbol{\psi}_{9}$ and $\boldsymbol{\psi}_{12}$ are very similar to linear eigenmodes of transverse beam vibration. In addition, KL decomposition of the simulated motion of the DEM model also reveils symmetric and asymmetric forms of combined transversal and longitudinal motion, as captured by characteristic functions $\psi_{3}, \psi_{7}, \psi_{10}$ and $\psi_{11}$.

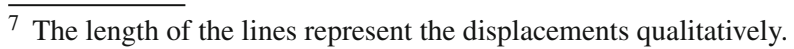

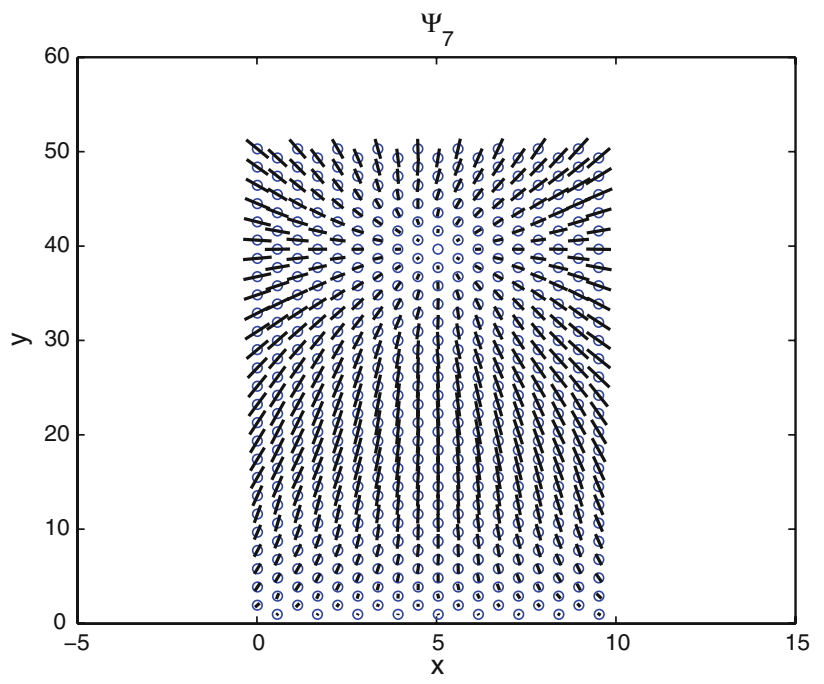

Fig. 15 Characteristic function $\psi_{7}$

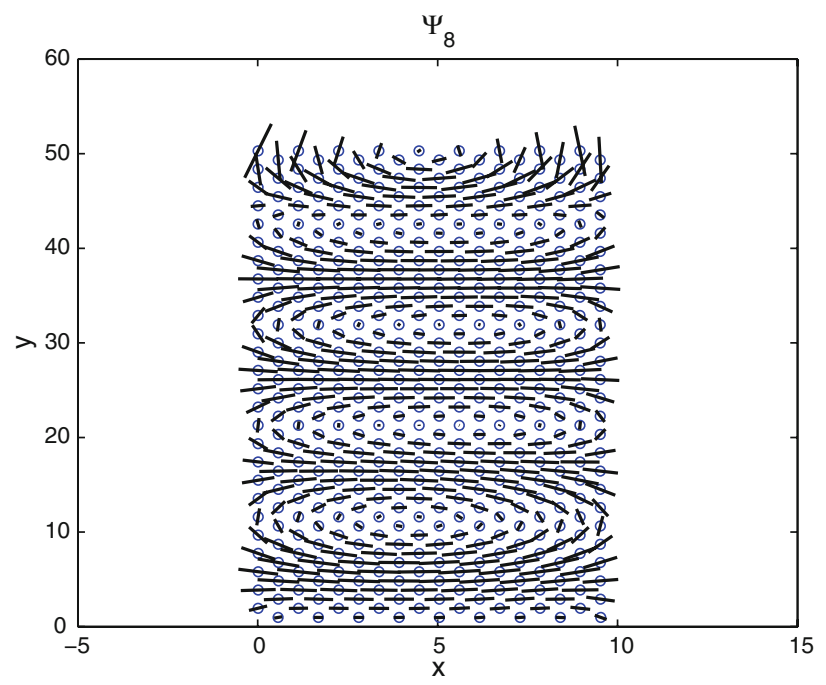

Fig. 16 Characteristic function $\psi_{8}$

\subsection{Reduced order hybrid DEM/KLT model}

As mentioned above, we keep the 234 most important particles as free particles $p_{i}^{\Theta}$ and choose basis $\tilde{\boldsymbol{\Psi}}=\left[\boldsymbol{\psi}_{1} \boldsymbol{\psi}_{2} \ldots\right.$ $\left.\psi_{19}\right]$ of the first 19 characteristic functions, derived from data set $\mathcal{D}$ as ansatz functions to model the dynamics of the 468 cluster particles $p_{i}^{\Gamma}$. Thus, instead of initially 1404 dof of the original DEM model, we now have a reduced hybrid DEM/KLT model of $468+19=487$ dof. We achieve a model order reduction by almost $2 / 3$.

Accuracy and sensitivity of reduced order hybrid DEM/KLT model

To compare the simulation results of the reduced order hybrid DEM/KLT model with the initial DEM model, we employ 


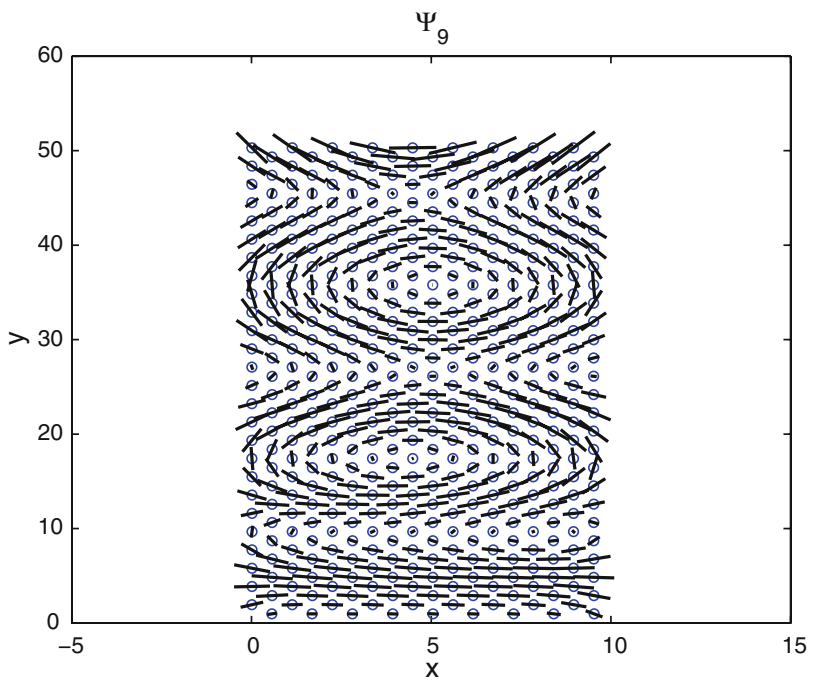

Fig. 17 Characteristic function $\boldsymbol{\psi}_{9}$

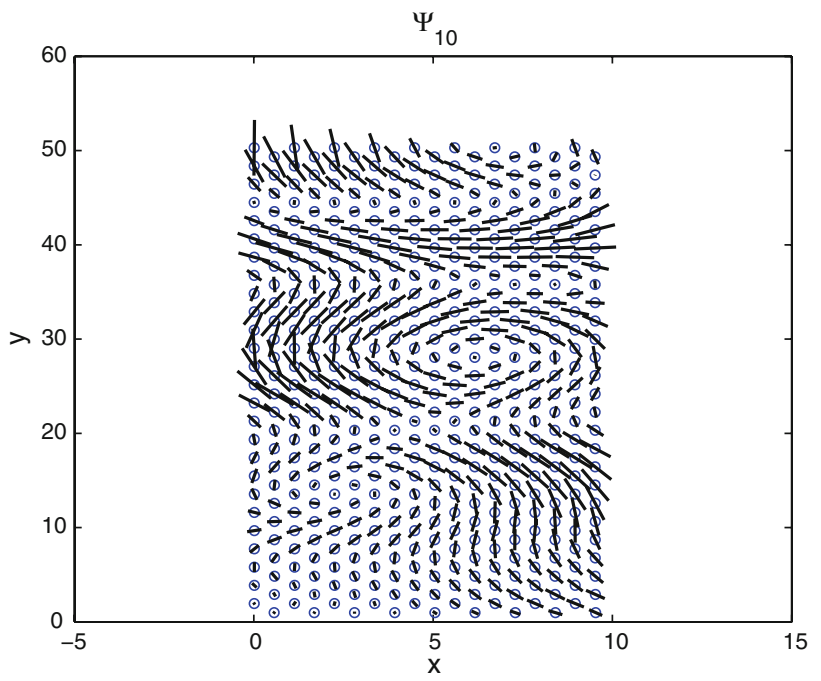

Fig. 18 Characteristic function $\psi_{10}$

performance functional

$\mathcal{J}_{e}=\frac{1}{N T} \sum_{n=1}^{T}\|\mathbf{q}(n)-[\tilde{\mathbf{q}}(n)+\overline{\mathbf{q}}]\| h$

as a measure, where $h$ is the step size of the numerical integration.

Since it is a well known fact that characteristic functions are case sensitive to changes in the system boundary conditions, we investigate the performance of the reduced basis of ansatz functions $\tilde{\boldsymbol{\Psi}}$ simulating the dynamics of the cantilever beam applying different forms of excitation.

Table 1 provides an overview of the simulation settings and results. It is obvious, that the reduced order hybrid DEM/ KLT model gives equally accurate results for all forms excitation while consuming only about $1 / 3$ of simulation time

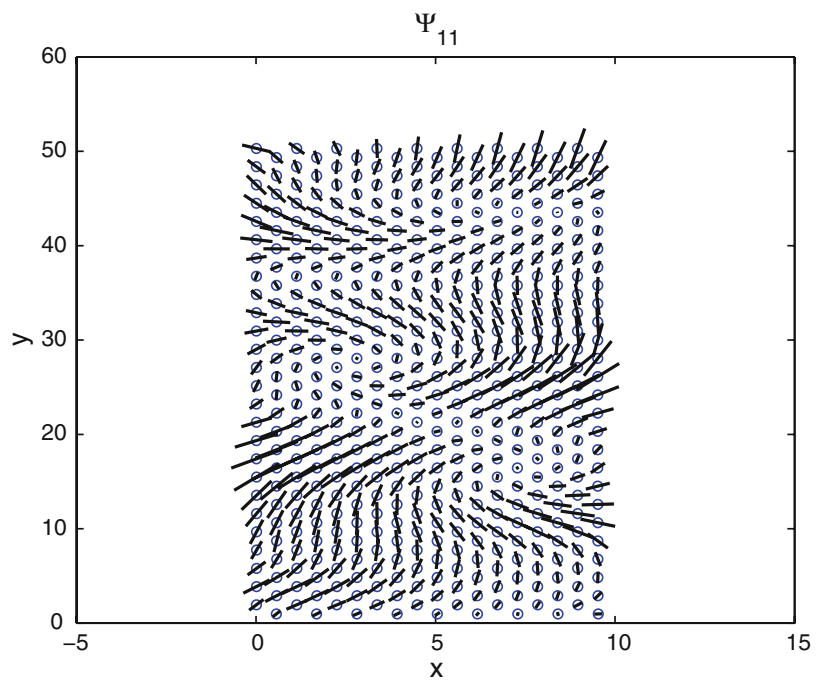

Fig. 19 Characteristic function $\psi_{11}$

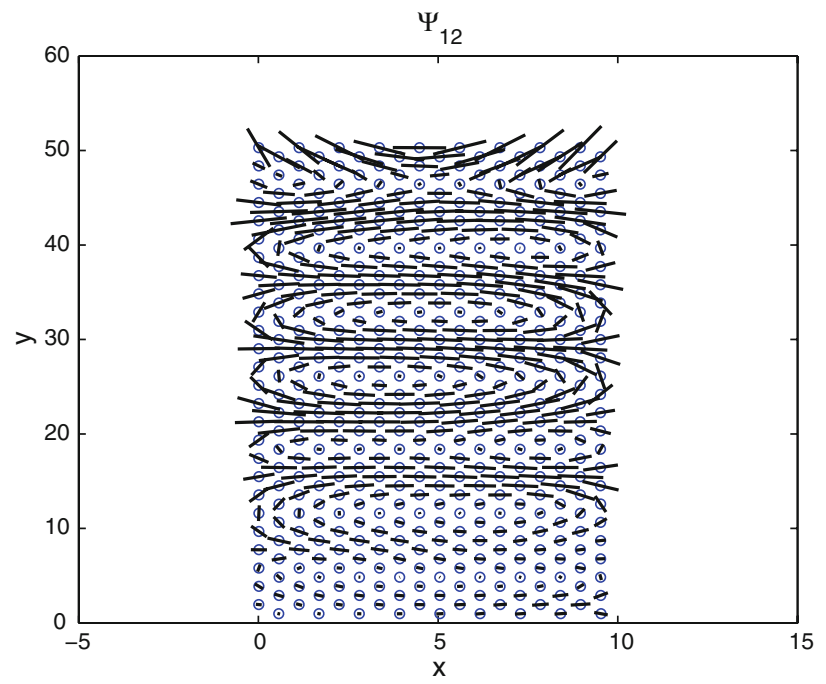

Fig. 20 Characteristic function $\boldsymbol{\psi}_{12}$

compared to the original DEM model. Naturally, there are limits to this procedure: These good approximations hold under moderate excitation amplitudes, only. At $0.4 \mathrm{~Hz}$ and at $0.8 \mathrm{~Hz}$ the cantilever beam shows resonance phenomena, resulting in larger vibrational amplitudes. In these cases, due to increased particle-particle distances the linear approximations of the Lennard-Jones particle forces become significantly inaccurate. Thus, in order to simulate larger particle displacements by reduced order hybrid DEM/KLT model it is be necessary, to approximate Lennard-Jones forces by nonlinear functions, as proposed in Sect. 4.6. 


\section{Conclusion}

The DEM is a well established procedure in molecular dynamics and recently, becomes more and more popular in structural mechanics. However, high computational costs still limit DEM applications for nano-scale mechanical problems.

In this paper, we introduce a new algorithm to approximate DEM models by reduced order hybrid DEM/KLT models. In an exemplary study we show reduction in model order and in simulation time of almost $2 / 3$ by the hybrid DEM/KLT method compared to the original, full-size DEM model.

The accuracy of the simulation results of the reduced order hybrid DEM/KLT model is surprisingly high considering the large reduction ratio in degrees of freedom. In this particular example, the choice of ansatz function for approximating the highly nonlinear Lennard-Jones particle-particle forces imposes limitations.

Nevertheless, we think that the reduced order hybrid DEM/

KLT model approach may be a milestone on the path towards atom-based nano-scale mechanical simulations.

Acknowledgments Author P. Glösmann is grateful for the support of Deutsche Forschungsgemeinschaft (DFG) under contract G1637/1-1.

Open Access This article is distributed under the terms of the Creative Commons Attribution Noncommercial License which permits any noncommercial use, distribution, and reproduction in any medium, provided the original author(s) and source are credited.

\section{References}

1. Cundall PA (1971) A computer model for simulation of progressive, large-scale movements in blocky rock. In: Proceedings of the ISRM-Symposium, Nancy, France 2:129-136

2. Schwager T, Pöschel T (2003) Rigid body dynamics of railway ballast. In: Popp K, Schiehlen W (eds) System dynamics and longterm behaviour of railway vehicles, track and subgrade. Springer, Berlin, pp 451-470

3. Karrech A, Duhamel D, Bonnet G et al (2007) A computational procedure for the prediction of settlement in granular materials under cyclic loading. Comput Methods Appl Mech Eng 197(1-4): 80-94

4. Lobo-Guerrero S, Vallejo LE (2007) Influence of pile shape and pile interaction on the crushable behavior of granular materials around driven piles: DEM analyses. Granular Matter 9(3):241250

5. D'Addetta GA, Kun F, Ramm E (2002) On the application of a discrete model to the fracture process of cohesive granular materials. Granular Matter 4(2):77-90

6. Egholm DL (2007) A new strategy for discrete element numerical models: 1. Theory J Geophys Res 112:B05203

7. Vesga LF, Vallejo LE, Lobo-Guerrero S (2008) DEM analysis of the crack propagation in brittle clays under uniaxial compression tests. Int J Numer Anal Methods Geomech 32(11):1405-1415
8. Alkhaldi H, Eberhard P (2007) Particle screening phenomena in an oblique multi-level tumbling reservoir: a numerical study using discrete element simulation. Granular Matter 9(6):415-429

9. Sykut J, Molenda M, Horabik J (2008) DEM simulation of the packing structure and wall load in a 2-dimensional silo. Granular Matter 10(4):273-278

10. Tano KT, Pålsson BI (2008) Assessment of mill lifter bar deflection measurements using wavelets and discrete element methods. Granular Matter 10(4):279-283

11. Fleissner F, Gaugele T, Eberhard P (2007) Applications of the discrete element method in mechanical engineering. Multibody Syst Dyn 18(1):81-94

12. Zang MY, Lei Z, Wang SF (2007) Investigation of impact fracture behavior of automobile laminated glass by $3 \mathrm{D}$ discrete element method. Comput Mech 41(1):73-83

13. Wang G, Ostoja-Starzewski M, Radziszewski P et al (2006) Particle modeling of dynamic fragmentation. II. Fracture in single- and multi-phase materials. Comput Mater Sci 35(2):116-133

14. Carmona HA, Kun F, Andrade JS et al (2007) Computer simulation of fatigue under diametrical compression. Phys Rev E 75:046115

15. Kim H, Wagoner MP, Buttlar WG (2008) Simulation of fracture behavior in asphalt concrete using a heterogeneous cohesive zone discrete element model. J Mater Civil Eng 20:552-563

16. Iordanoff I, Battentier A, Neauport J et al (2008) A discrete element model to investigate sub-surface damage due to surface polishing. Tribol Int 41(11):957-964

17. Meguro K, Tagel-Din HS (2002) Applied element method used for large displacement structural analysis. J Nat Deasaster Sci 24(1):25-34

18. Raje N, Sadeghi F, Rateick RG (2007) A discrete element approach to evaluate stresses due to line loading on an elastic half-space. Comput Mech 40(3):513-529

19. Liu WK, Karpov EG, Zhang S et al (2004) An introduction to computational nanomechanics and materials. Comput Methods Appl Mech Eng 193:1529-1578

20. Tavarez FA, Plesha ME (2007) Discrete element method for modelling solid and particulate materials. Int J Numer Methods Eng 70(4):379-404

21. Azevedo NM, Lemos JV (2006) Hybrid discrete element/finite element method for fracture analysis. Comput Methods Appl Mech Eng 195(33-36):4579-4593

22. Griebel M, Zumbusch G, Knapek S et al (2007) Numerical simulations in molecular dynamics. Springer, Berlin

23. Pöschel T, Schwager T (2005) Computational granular dynamics. Springer, Berlin

24. Zohdi TI (2005) Charge-induced clustering in multifield particulate flows. Int J Numer Methods Eng 62(7):870-898

25. Meyer M, Matthies HG (2003) Efficient model reduction in nonlinear dynamics using the Karhunen-Loève expansion and dualweighted-residual methods. Comput Mech 31:179-191

26. Lall S, Krysl P, Marsden JE (2003) Structure-preserving model reduction for mechanical systems. Phys D 184:304-318

27. Glösmann P, Kreuzer E (2005) Nonlinear system analysis with Karhunen-Loève transform. Nonlinear Dyn 41(1-3):111-128

28. Wang F, Bajaj AK (2007) Nonlinear normal modes in multi-mode models of an inertially coupled elastic structure. Nonlinear Dyn 47:25-47

29. Karhunen K (1946) Zur Spektraltheorie stochastischer Prozesse. Ann Acad Sci Fen 34

30. Sirovich L (1987) Turbulence and the dynamics of coherent structures. Q Appl Math 45(3):561-582

31. Glösmann P, Kreuzer E (2009) On the application of KarhunenLoève transform to transient dynamic systems. J Sound Vib. doi:10. 1016/j.jsv.2009.08.013 